\title{
The Optimal Degree of Gravity Spherical Harmonic Model Calculation for Gravity Disturbance Compensation in Inertial Navigation
}

\author{
Junbo TIE ${ }^{1}$, Meiping WU ${ }^{1}$, Juliang Cao ${ }^{1}$, Junxiang Lian ${ }^{1}$, Shaokun Cai ${ }^{1}$ and Lin Wang ${ }^{1}$ \\ ${ }^{1}$ National University of Defense Technology, Changsha 410073, People's Republic of China
}

\begin{abstract}
In recent years, the significant improvement of inertial navigation, leaves the gravity disturbance as the important factor which affects the accuracy of inertial navigation. This paper focus on the compensation for gravity disturbance with gravity spherical harmonic model, especially the optimal degree of gravity spherical harmonic model with which to calculate the gravity disturbance. The effect of gravity disturbance on inertial navigation is analysed based on the amplitude-frequency response characteristics of inertial navigation error differential equation, then the dominantly influential frequency band of gravity disturbance can be found which is the target of compensation. Combination the dominantly influential frequency band with the spatial resolution of the Earth's gravity spherical harmonic model EGM2008 which is used to calculate the gravity disturbance, the optimal degree can be determined based on an algorithm proposed in this paper. Finally, shipborne inertial navigation experiment confirms the correctness and effectiveness of the proposed algorithm.
\end{abstract}

\section{Introduction}

Inertial navigation system (INS) is currently the main means of navigation which measures the vehicle's motion and constantly updates position. INS is based on Newton's Second Law, which combines specific force and gravity into acceleration. The velocity and position can be obtained through integration of acceleration. Specific force can be measured by inertial sensors, three accelerometers of INS, and the gravity information is acquired from the normal gravity model.

The performance of INS depends not only on the quality of inertial sensors, but also on the accuracy of the gravity information $[1,2]$. In the limiting situation of drift-free gyroscopes and perfect accelerometers, INS still cannot be error-free, because uncertainties in the normal gravity model will produce errors. In recent years, the significant improvement of INS, especially on inertial sensors, leaves gravity disturbance as the important error source of the navigation solution, particularly for rough topological areas $[4,5]$. For future higher precision navigation, the compensation of gravity disturbance has to be considered [10].

Nowadays, with the release of ultra-high degree Earth Gravitational Model, such as Earth Gravitational Model 2008 (EGM2008) [6, 7], the gravity disturbance on the vehicle's trajectory can be calculated directly which can be used to compensate the effect of gravity disturbance on INS. EGM2008 is publicly released by the U.S. National Geospatial Intelligence Agency (NGA) EGM Development Team. This gravitational model is a spherical harmonic model (SHM) and is complete to spherical harmonic degree and order 2159, and contains additional coefficients extension to degree 2190 and order 2519 and the full access to this model's coefficients is provided in [3]. It is because EGM2008 has degree and order up to 2190 that the gravity disturbance can be precisely calculated with this model. However, the high degree and order bring a great computation burden on the embedded navigation computer of INS in real time application. [4] indicates the total operation count for calculating spherical model. To reduce the computation complexity and maintain the compensation accuracy, the interest lies in this paper is to find an optimal degree used in the calculation of EGM2008 instead of the maximum degree 2190.

The paper is organized as follows: in section 2, the effect of gravity disturbance on INS is analysed to determine the dominantly influential frequency band of gravity disturbance. Then the calculation of SHM is introduced in section 3 and the algorithm of determining the optimal degree is proposed in section 4. The shipborne inertial navigation experiment is in section 5, and the conclusion is provided in section 6 .

\section{Effect of Gravity Disturbance on INS}

To find the dominantly influential frequency band of gravity disturbance, the error characteristics of gravity disturbance induced error is investigated. The north channel of INS is analyzed here and the only error source considered is the gravity disturbance, and the error differential equation are as follow [8-10].

$$
\delta \dot{\theta}=-\delta v_{N} / R
$$




$$
\begin{gathered}
\delta \dot{v}_{N}=g \delta \theta+\delta g \\
\delta \dot{p}=\delta v_{N}
\end{gathered}
$$

where $\delta \theta$ is the pitch error, $\delta v_{N}$ is the north velocity error, $R$ is the mean radius of the Earth, $\delta p$ is the position error, $g$ is the normal gravitational acceleration, and $\delta g$ is the input error source, the gravity disturbance.

These error equations can be described with state equation. According to the theory of linear system, the transfer function can be obtained from by the following transformation [11].

$$
\boldsymbol{G}(s)=\left[\boldsymbol{C}(s \boldsymbol{I}-\boldsymbol{F})^{-1} \boldsymbol{B}\right]
$$

The transfer function can be obtained as follow

$$
\boldsymbol{G}(s)=R /\left(R s^{2}+g\right)
$$

The amplitude-frequency response of the north channel of INS can be obtained based on the transfer function, equation (5).

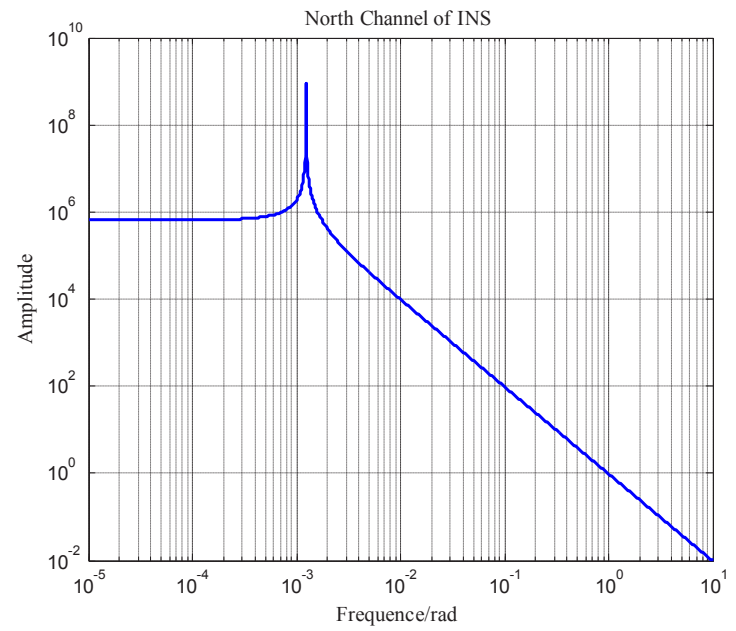

Figure 1. The amplitude-frequency response of the north channel of INS

It can be seen that the response of INS for gravity disturbance is alike a low-pass filter, and the cut-off frequency can be determined according to the $-3 \mathrm{~dB}$ definition, the cut-off frequency $f_{\text {cut-off }}$ is $0.00215 \mathrm{rad}$ or $3.42 \times 10^{-4} \mathrm{~Hz}$, and the peak of the amplitude-frequency response is the Schuler frequency of INS. Because of the low-pass characteristic, the high frequency gravity disturbance is filtered out, and only the low frequency gravity disturbance will have a negative effect on the INS. Therefore, the dominantly influential frequency band of gravity disturbance is determined.

$$
f_{\text {compen }} \leq 3.42 \times 10^{-4} \mathrm{~Hz}
$$

\section{Gravity Disturbance and Gravity Spherical Harmonic Model}

The gravity disturbance means the difference between the gravity vector calculated through the normal gravity model (NGM) and the actual one, including difference in magnitude and direction [12]. The difference in magnitude is the so-called gravity anomaly, and the difference in direction is the so-called deflection of vertical (DOV) which is the chief cause of the gravity- induced errors [13-15]. As shown in figure 2, DOV is the angle between the true plumb line and the normal line of the reference ellipsoid which is chosen to approximate the Earth's sea-level surface. DOV has a north component $x$ and an east component $h$, the order of DOV component can maximally reach 100 arcsec which is significantly higher than the modern accelerometer bias, whose typical value is $10 \mathrm{mGal}\left(1 \mathrm{mGal}=10^{-5} \mathrm{~m} / \mathrm{s}^{2}\right)$ [4], corresponding to a $2 \operatorname{arcsec}$ DOV [3]. The horizontal gravity disturbance, including north component $\delta g_{N}$ and east component $\delta g_{E}$, are caused by DOV as follow.

$$
\delta g_{N}=g \cdot \xi \quad \delta g_{E}=g \cdot \eta
$$

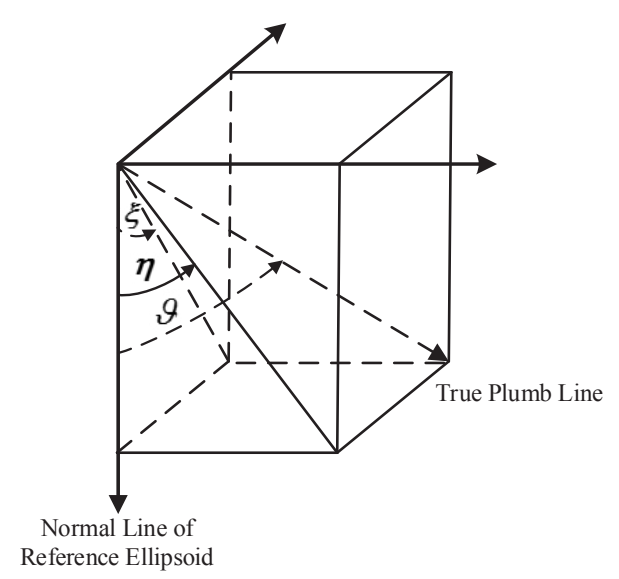

Figure 2. The definition of DOV

The horizontal gravity disturbance can be calculated based on the gravity spherical harmonic model $[3,16]$

$$
\delta g_{N}=-\frac{G M}{r^{2}} \sum_{n=2}^{\infty}\left(\frac{a}{r}\right)^{n} \sum_{m=0}^{n}\left(\begin{array}{c}
\bar{C}_{n m}^{*} \cos m \lambda \\
+\bar{S}_{n m} \sin m \lambda
\end{array}\right) \frac{d \bar{P}_{n m}(\cos \theta)}{d \theta}
$$

$\delta g_{E}=-\frac{G M}{r^{2} \sin \theta} \sum_{n=2}^{\infty}\left(\frac{a}{r}\right)^{n} \sum_{m=0}^{n} m\left[\begin{array}{l}\bar{C}_{n m}^{*}(-\sin m \lambda) \\ +\bar{S}_{n m} \cos m \lambda\end{array}\right] \bar{P}_{n m}(\cos \theta)$

where $G$ is Newton's gravitational constant, $M$ is the mass of the Earth, $r$ is the geocentric radius distance, $\theta$ is the polar distance, $\bar{C}_{n m}^{*}$ and $\bar{S}_{n m}$ are coefficients of the spherical harmonic of degree $n$ and order $m$, $\bar{P}_{n m}(\cos \theta)$ is the normalized associated Legendre function of the first kind. The Legendre function can be resolved by recursion[17].

\section{The Algorithm of Choosing Optimal Degree}

In Section 2, the dominantly influential frequency band of gravity disturbance has been determined. However, gravity disturbance is a spatial-domain signal rather than a time-domain signal, and the gravity disturbance signal comes into INS as the position of INS changing on the 
surface of the Earth, hence the gravity disturbance signal can be transformed from spatial-domain into time-domain with the velocity of INS, and the associated dominantly influential frequency band can also be transformed into spatial domain as

$$
f_{\text {compen }}^{\prime}=\frac{f_{\text {compen }}}{v}
$$

where $v$ is the velocity of INS, and $f_{\text {compen }}^{\prime}$ is the compensation frequency band in spatial-domain, and the corresponding period of the compensation frequency band which is a spatial distance can be determined

$$
D_{\text {compen }}=\frac{1}{f_{\text {compen }}^{\prime}}=\frac{v}{f_{\text {compen }}}
$$

According to the property of spherical harmonic function, the spatial distance $D_{\text {compen }}$ is determined by the degree of the SHM. Assume that the degree used in SHM calculation is $n$, then the sphere of the Earth is divided by the Legendre function into $n$ zones, so the spatial resolution of degree $n \mathrm{SHM}$ is

$$
D=\frac{\pi R}{n}
$$

Substituting equation (12) into equation (11), the optimal degree of gravity spherical harmonic model can be obtained.

$$
n_{\text {compen }}=\frac{\pi R f_{\text {compen }}}{v}
$$

There are some special cases need to be considered, the first is that the maximum of $n_{\text {compen }}$ is 2190 when the used gravity spherical harmonic model is EGM2008. Second, if the velocity is very small, even zero, then assume $n_{\text {compen }}$ equal to 2190 is appropriate.

\section{The Shipborne Inertial Navigation Experiment}

The shipborne experiment instruments consists of a highprecision strapdown inertial navigation system (SINS) as shown in figure 4 and a $\operatorname{Novatel}^{\circledR}$ GPS receiver contained in the electric system of SINS.

The inertial sensors used in SINS are high precision ring laser gyros and quartz flexible accelerometers. The sampling rate of SINS is $200 \mathrm{~Hz}$ and the sample rate of GPS receiver is $2 \mathrm{~Hz}$, and the GPS data is processed off line with precise point positioning (PPP) technology to obtain position results as the reference in this experiment. The ship was first at moor, then sailed in maneuver, and the experiment time is 24 hours. The DOV values on the sailing trajectories are calculated based on EGM2008, and the position information used to calculate DOV is from GPS solution, and the period of calculating gravity disturbance with SHM is 100 seconds.

There are three types method to deal with the inertial navigation date: processing the inertial navigation data without compensation for gravity disturbance is type $\mathbf{I}$. Processing the data with compensation where the degree of SHM is set to 2190 is type II, and if the degree of
SHM is optimally set based on the proposed algorithm in section 4, that's type III.

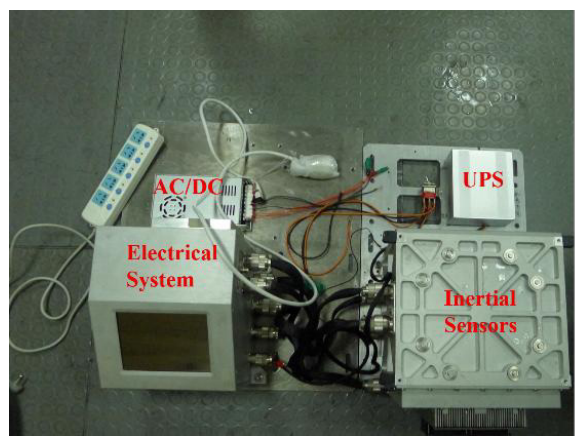

Figure 4. High precision strapdown inertial navigation system

The comparison of north component of gravity disturbance on the trajectory between type II and type III is shown in figure 5, and the comparison of east component of gravity disturbance on the trajectory between type II and type III is shown in figure 6 .

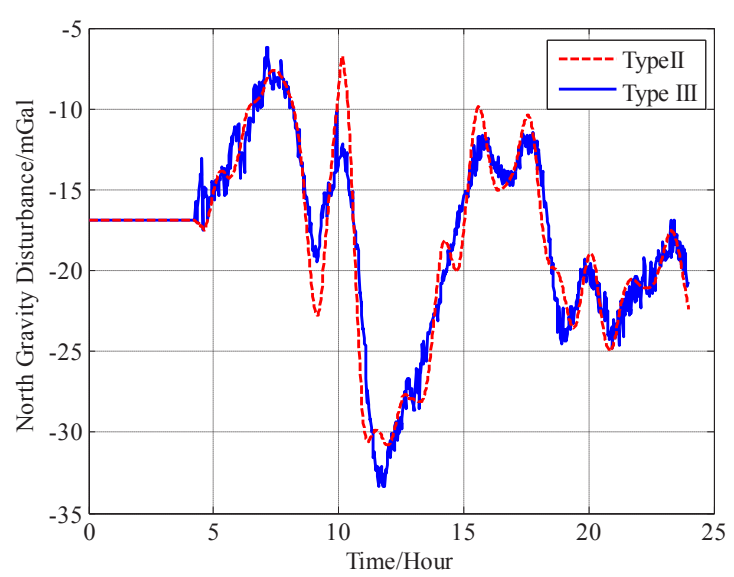

Figure 5. The comparison of north component of gravity disturbance on the trajectory

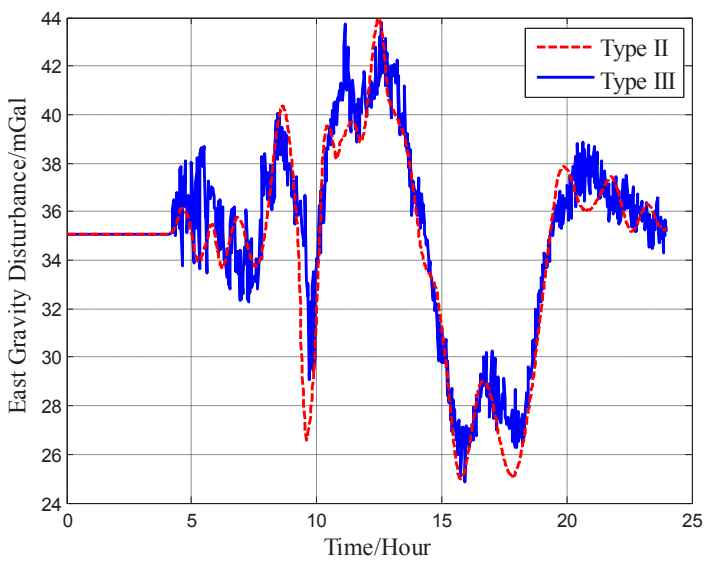

Figure 6. The comparison of east component of gravity disturbance on the trajectory

The comparison of time-consuming between type II and type III is shown in Table 1. With the optimal degree, the time for calculating SHM decreases by 635.8 seconds, 
about $36.1 \%$. From figure 7 , it can be seen that the average one-time time consuming of calculating SHM with full degree 2190 in type II is about 2 second. As for Type III, in the first five hours, the experiment ship is at moor, the velocity almost equals to zero, so the optimal degree is 2190 , after that the experiment ship begins to move, and the optimal degree is decreased to 1400 as shown in figure 8 , then the time-consuming is shortened to about 1 second.

Table 1. Comparison of time-consuming between type II and type III

\begin{tabular}{|c|c|c|}
\hline $\begin{array}{c}\text { Time-consuming Item } \\
\text { /Second }\end{array}$ & Type II & Type III \\
\hline Total time-consuming & 3542.1 & 2938.3 \\
\hline $\begin{array}{c}\text { Time for navigation } \\
\text { calculation }\end{array}$ & 1722.5 & 1748.6 \\
\hline Time for calculating SHM & 1762.4 & 1126.6 \\
\hline $\begin{array}{c}\text { Time for initial alignment and } \\
\text { others }\end{array}$ & 57.2 & 63.1 \\
\hline $\begin{array}{c}\text { Decrease of total time- } \\
\text { consuming }\end{array}$ & 603.8 \\
\hline $\begin{array}{c}\text { Decrease of time for } \\
\text { calculating SHM }\end{array}$ & 635.8 \\
\hline
\end{tabular}

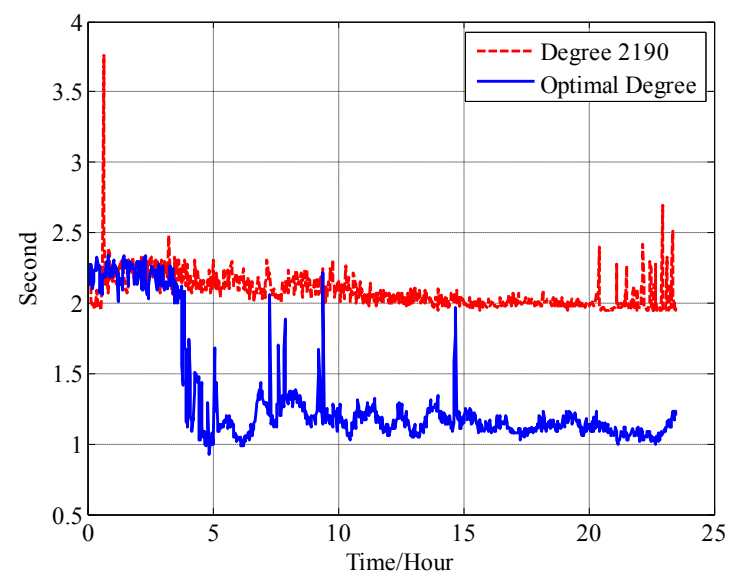

Figure 7. The time-consuming for one-time calculating SHM in type II and type III

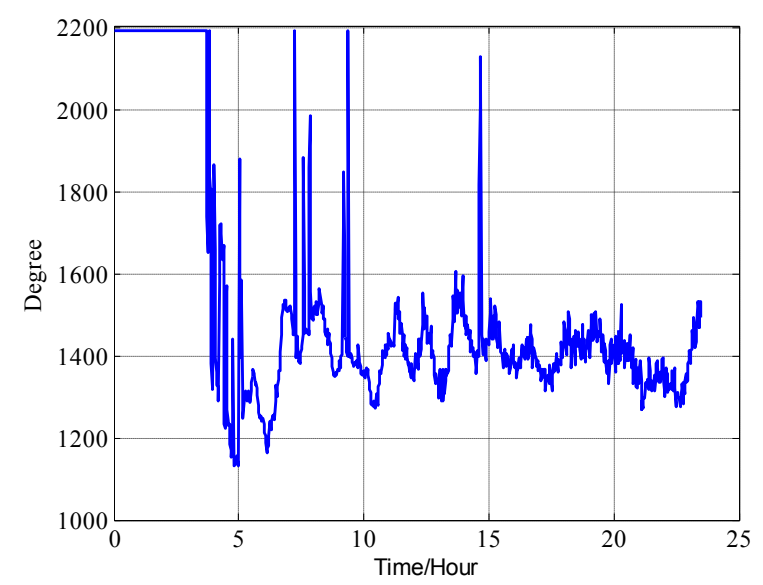

Figure 8. The optimal degree of calculating SHM in type III
The positioning error comparison among the three types are shown in figure 12 , it can been seen that the position errors of type II and type III are both less than type I, which means the compensation for gravity disturbance is efficient. Subtracting the position error of type II and type III from Type I, the improvement of positioning accuracy can be obtained, as shown in figure 10 , and it can be seen that the precision improvement with optimal degree is almost consistent with the full degree. Attention is needed here, the vibration of precision improvement of type III is obviously smaller than that of type II, this is probably because that there exists some error in the high degree components of the SHM, and these errors is avoided by utilization of the optimal degree.

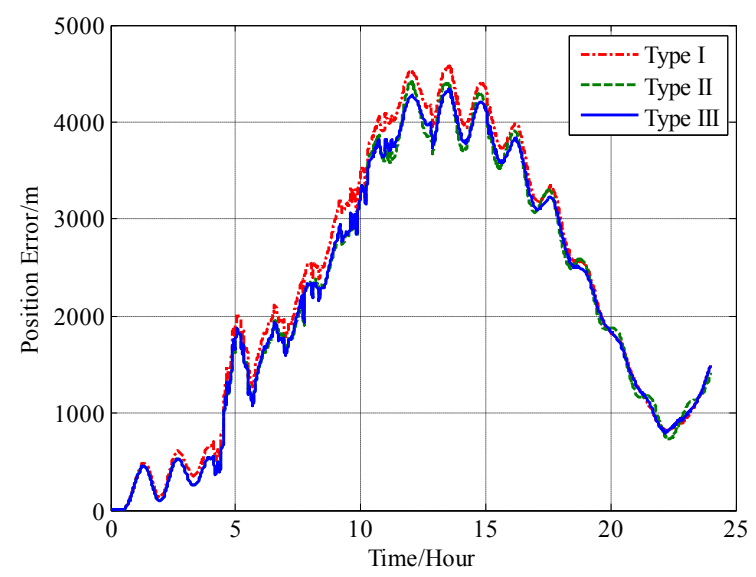

Figure 9. The comparison of position error

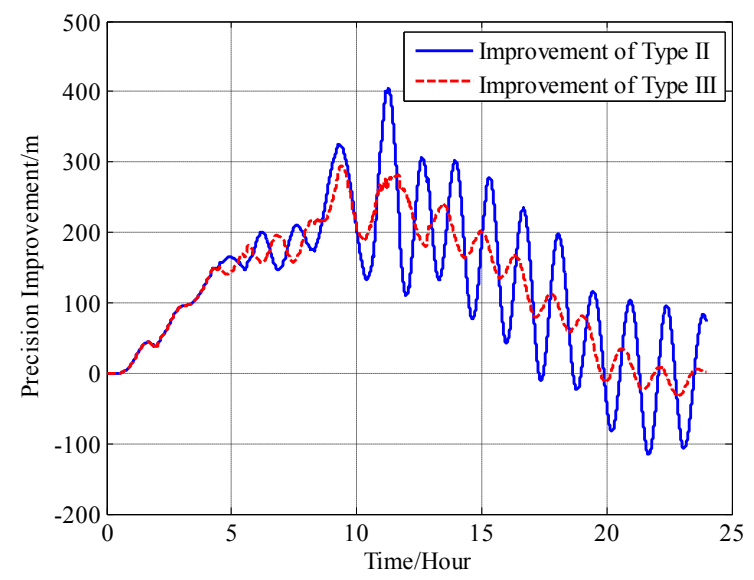

Figure 10. The comparison of position error

\section{Conclusion}

In this paper, the optimal degree of calculating SHM for gravity disturbance compensation is discussed. First, the effect of gravity disturbance on inertial navigation is analysed based on the amplitude-frequency response characteristics of inertial navigation error differential equation. Second, the dominantly influential frequency band of gravity disturbance is determined which is the target of compensation. Then combination the dominantly influential frequency band with the spatial resolution of the Earth's gravity spherical harmonic model, the algorithm of determining the optimal degree 
with which to implement the SHM calculation is proposed. Finally, the shipborne inertial navigation experiment confirms the correctness and effectiveness of the proposed algorithm, with the optimal degree the timeconsuming of calculating SHM decreases by $36.1 \%$, and the efficiency of DOV compensation is almost consistent with that of full degree.

\section{Acknowledgment}

This work is supported by the National Key R\&D Program of China, contract No. 2016YFC0303002; and National Natural Science Foundation of China, contract No. 61603401.

\section{References}

1. Kwon $\mathrm{J} \mathrm{H}$ and Jekeli $\mathrm{C}$ Gravity requirements for ultra-precise inertial navigation J. Navig. 58 479-92 (2005)

2. David W. Harriman and J. Chris Harrison GravityInduced Errors in Airborne Inertial Navigation J.Guidance. 9 419-426 (1986)

3. URL of EGM2008: http://earthinfo.nga.mil/GandG/wgs84/gravitymod/egm2008

4. Jing W, Gongliu Y, Xiangyun L and Zhou X Application of the spherical harmonic gravity model in high precision inertial navigation systems Meas. Sci. Technol. 27095103 (2016)

5. Zhou X, Gongliu Yang, Jing $\mathrm{W}$ and Jing L An improved gravity compensation method for highprecision free-INS based on MEC-BP-AdaBoost Meas. Sci. Technol. 27125007 (2016)

6. Pavlis N. K., Holmes S. A., Kenyon S. C. and Factor J. K. An Earth Gravitational Model to Degree 2160: EGM2008. In Proceedings of the European Geosciences Union General Assembly 2008, Vienna, Austria, 13-18 (2008)

7. Pavlis N. K., Holmes S. A., Kenyon S. C. and Factor J. K. The development and evaluation of the Earth Gravitational Model 2008 (EGM2008) J. Geophys. Res. Solid Earth 117 1-38 (2012)

8. D. Titterton and J. Weston, Strapdown Inertial Navigation Technology. (U.K.: The Institution of Electrical Engineers 2004)

9. Paul D. Groves Principles of GNSS, Inertial, and Multisensor Integrated Navigation Systems (London: Artech House 2008)
10. Jekeli C Precision free-inertial navigation with gravity compensation by an onboard gradiometer Journal of Guidance, Control and Dynamic. 29704 13 (2006)

11. Chi-Tsong Chen. Linear System Theory Design (Oxford University Press, USA 1998)

12. Wellenhof B H and Moritz H Physical Geodesy 2nd edn (Graz: Springer 2005)

13. Xiao Z., Gongliu Y., Qingzhong C. and Jing W. A novel gravity compensation method for high precise free-ins based on extreme learing machine Sensors. 162019 (2016)

14. Ruonan W, Qiuping W, Fengtian H, Tianyi L, Peida $\mathrm{H}$ and Haixia $\mathrm{L}$ Gravity Compensation Using EGM2008 for High-Precision Long-Term Inertial Navigation Systems Sensors. 162177 (2016)

15. Jing W, Gongliu Y An Online Gravity Modeling Method Applied for High Precision Free-INS. Sensors 161541 (2016)

16. Jekeli C, Lee J K and Kwon J H 2007 On the computation and approximation of ultra-high-degree spherical harmonic series [J]. Journal of Geodesy, 81 603-615 (2007)

17. Wei Zi-Qing. Recurrence Relations for Fully Normalized Associated Legendre Functions and Their Derivatives and Integrals. Geomatics and Information Science of Wuhan University, 41 27-36 (2016)

18. Jiancheng Fang, LinZhouting Chen, and Jifeng Yao. An Accurate Gravity Compensation Method for High-Precision Airborne POS. IEEE Transactions on Geoscience and Remote Sensing, 52 (2014)

19. Dai D, Wang X, Zhan D and Huang Z. An improved method for dynamic measurement of deflections of the vertical based on the maintenance of attitude reference. Sensors, 14, 16322 (2014)

20. Siouris G M. Gravity modeling in aerospace applications. Aerospace Science and Technology, 13 301-315 (2009)

21. Gleason D. Critical Role of Gravity Compensation in a Stand-Alone Precision INS. DARPA PINS Meeting, Arligton, Virginia (2003)

22. Jekeli C. Gravity on Precise Short-Term, 3-D Free Inertial Navigation. Navigation, 44 347-357 (1997) 